\title{
Bulk Aluminum at High Pressures: A First-Principles Study
}

\author{
Michael J. Tambe, Nicola Bonini, and Nicola Marzar: \\ Department of Materials Science and Engineering, \\ Massachusetts Institute of Technology, Cambridge MA 02139
}

\begin{abstract}
The behavior of metals at high pressure is of great importance to the fields of shock physics, geophysics, astrophysics, and nuclear materials. We study here bulk crystalline aluminum from firstprinciples at pressures up to $2500 \mathrm{GPa}$ - soon within reach of laser-based experimental facilities. Our simulations use density-functional theory and density-functional perturbation theory in the localdensity and generalized-gradient approximations. Notably, the two different exchange-correlation functionals predict very similar results for the $f c c \rightarrow h c p, f c c \rightarrow b c c$, and $h c p \rightarrow b c c$ transition pressures, around $175 \mathrm{GPa}, 275 \mathrm{GPa}$, and $380 \mathrm{GPa}$ respectively. In addition, our results indicate that core overlaps become noticeable only beyond pressures of $1200 \mathrm{GPa}$. From the phonon dispersions of the fcc phase at increasing pressure, we predict a softening of the lowest transverse acoustic vibrational mode along the [110] direction, which corresponds to a Born instability of the fcc phase around $725 \mathrm{GPa}$.
\end{abstract}

First-principles calculations have proved useful to the

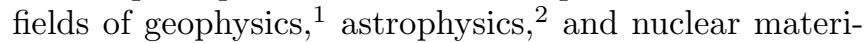
als $\underline{\underline{3}}$ Aluminum, being cubic close-packed and having no d-shell electrons, is a prototype for theoretical predictions and understanding the high-pressure behavior of

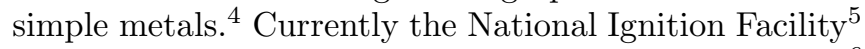
at LLNL is expected to achieve shockless compression ${ }^{6}$ of metals up to $2000 \mathrm{GPa}$. This new facility may provide rapid advancements to high-pressure physics and could partner very successfully with theoretical studies.

The equation of state (EOS) and phase stability of aluminum were first studied from first-principles in the early $1980 \mathrm{~s}, \frac{7,8,9}{7 n}$ all cases the predicted phase sequence was $f c c \rightarrow h c p \rightarrow b c c$, but predictions differed greatly in the transition pressures. Several other calculations within the local-density approximation (LDA) 10 or the generalized-gradient approximation (GGA) 11,12 have since then been performed, with a predicted static (i.e. without the phonon contribution) $f c c \rightarrow h c p$ transition pressure of $205 \pm 20 \mathrm{GPa}^{10}$ in $\mathrm{LDA}$ and $170 \mathrm{GPa} 11$ and $192 \mathrm{GPa} 2 \underline{12}$ in GGA. These discrepancies are more notable for the $h c p \rightarrow b c c$ transition pressure: $565 \pm 60 \mathrm{GPa}^{10}$ in LDA versus $360 \mathrm{GPa}^{11}$ in GGA, leaving significant uncertainties open. Theoretical work on the vibrational properties of aluminum also suggests for the $f c c \rightarrow h c p$ transition a transition pressure higher than the static one $\underline{11,12}$ Elastic properties $\frac{13,14}{14}$ and the absolute strength under tension 15 have also been calculated; the latter results are of particular interest as they demonstrate the important role vibrational modes play in determining mechanical stability and suggest that shear failure modes are inherent in aluminum.

Experimentally, the equation of state of aluminum at high pressures was studied by shock-compression 16 at pressures above the predicted maximum for the $f c c \rightarrow$ $h c p$ phase boundary, $\stackrel{10}{\rightleftharpoons}$ but a transition was not observed. However recent diamond anvil cell experiments observed a $f c c \rightarrow h c p$ transition at $217 \pm 10 \mathrm{GPa} 17$ highlighting the difficulty in achieving thermodynamic equilibrium in shock-compression.

In this article we report first-principles calculations of aluminum under hydrostatic compression up to 2500 GPa. In order to assess mechanical stability under shock, we also calculate the vibrational properties in the fcc phase and determine the elastic constants from the slopes of the phonon dispersions (i.e. the sound velocities).

The equations of state in the fcc, bcc, and hcp phases have been calculated with density-functional theory (DFT) within both $\mathrm{LDA}^{18}$ and GGA ${ }^{19}$ Calculations have used the QUANTUM-ESPRESSO package ${ }^{20} \mathrm{We}$ use plane-wave basis sets and pseudopotentials and both 3 electron (3e) norm-conserving pseudopotentials, $\stackrel{21}{\underline{1}}$ with the $3 \mathrm{~s}$ and $3 \mathrm{p}$ electrons in the valence and nonlinear corecorrections, and 11 electron (11e) ultrasoft pseudopotentials 22 where the $2 \mathrm{~s}$ and $2 \mathrm{p}$ electrons, usually frozen in the core, are included explicitly in the valence. The inclusion of the $2 \mathrm{~s}$ and $2 \mathrm{p}$ electrons in the valence is essential to investigate the relevance of inner core electrons at high pressure. The planewave cutoffs for the wavefunctions are 25 Ry and 100 Ry for the $3 \mathrm{e}$ and 11e pseudopotentials, respectively, and 150 Ry and 800 Ry for the charge density. Brillouin zone integrations have been performed using a cold smearing $\frac{14}{4}$ of 0.02 Ry over shifted Monkhorst-Pack meshes of order $16 \times 16 \times 16$ for the fcc, $22 \times 22 \times 22$ for the bcc and $16 \times 16 \times 10$ for the hcp phases. The large sizes of the k-point meshes are necessary to obtain fully-converged transition pressures. The data for the total energy as function of volume have been fitted to the Birch 3rd order $\operatorname{EOS}^{23}$ near equilibrium to obtain equilibrium volumes and bulk moduli. The data for the equation of state have been determined from calculations performed at around 50-100 different volumes over a pressure range of $0-2500 \mathrm{GPa}$. Finally, the vibrational properties have been computed using densityfunctional perturbation theory (DFPT) $\stackrel{24}{4}^{\text {The dynami- }}$ cal matrices have been calculated on a $4 \times 4 \times 4 q$-point mesh and Fourier interpolation has been used to evaluate the phonon frequencies on finer grids.

The role of the inner core electrons is of primary concern at very high pressures. Under normal conditions, there is not sufficient overlap between the core and valence shell electrons to question the frozen-core approxi- 
TABLE I: The equilibrium lattice parameters, bulk moduli, and transition pressures of $\mathrm{Al}$ calculated with the different pseudopotentials described in the text, and compared to experimental results.

\begin{tabular}{|llllll|}
\hline & $a_{0}[\AA]$ & $B_{0}[\mathrm{GPa}]$ & $f c c \rightarrow h c p$ & $f c c \rightarrow b c c$ & $h c p \rightarrow b c c$ \\
\hline 11e GGA & 4.044 & 73.2 & $175 \mathrm{GPa}$ & $275 \mathrm{GPa}$ & $383 \mathrm{GPa}$ \\
\hline 11e LDA & 3.985 & 83.7 & $172 \mathrm{GPa}$ & $272 \mathrm{GPa}$ & $380 \mathrm{GPa}$ \\
\hline 3e GGA & 4.055 & 71.8 & $180 \mathrm{GPa}$ & $285 \mathrm{GPa}$ & $420 \mathrm{GPa}$ \\
\hline Expt. & $4.025^{a}$ & $78.3^{a}$ & $217 \mathrm{GPa}^{b}$ & - & - \\
\hline
\end{tabular}

${ }^{a}$ Ref. 26

${ }^{b}$ Ref. 17

(a)

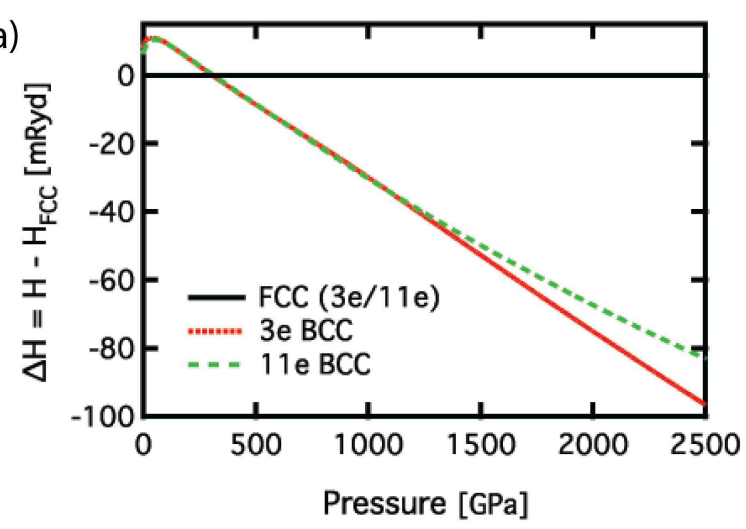

(b)

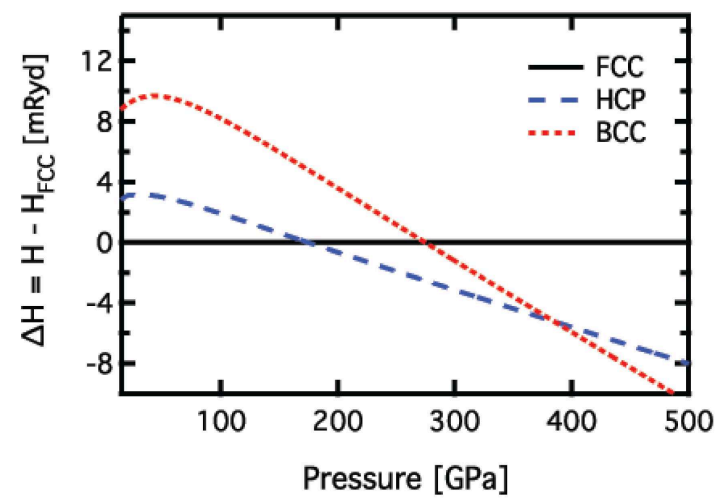

FIG. 1: (a) Enthalpy of the bcc phase (3e and 11e GGA) relative to the enthalpy of the fcc phase ( $3 \mathrm{e}$ and 11e GGA, respectively). (b) Enthalpies for hcp and bcc phase relative to the enthalpy of the fcc phase. The $f c c \rightarrow h c p, f c c \rightarrow b c c$, and $h c p \rightarrow b c c$ transition pressures are $175 \mathrm{GPa}, 275 \mathrm{GPa}$, and 380 GPa respectively.

mation,,$\underline{25}$ but at the pressures considered here core overlaps may become significant. To study the validity of the frozen-core approximation, we first compared the equations of state for different phases using both the $3 \mathrm{e}$ and 11e pseudopotentials. We report in Table I the equilibrium lattice parameters and bulk moduli in the fcc phase at zero pressure, and in Fig. 11 the relative enthalpies of fcc and bcc $\mathrm{Al}$ with respect to the fcc phase, up to
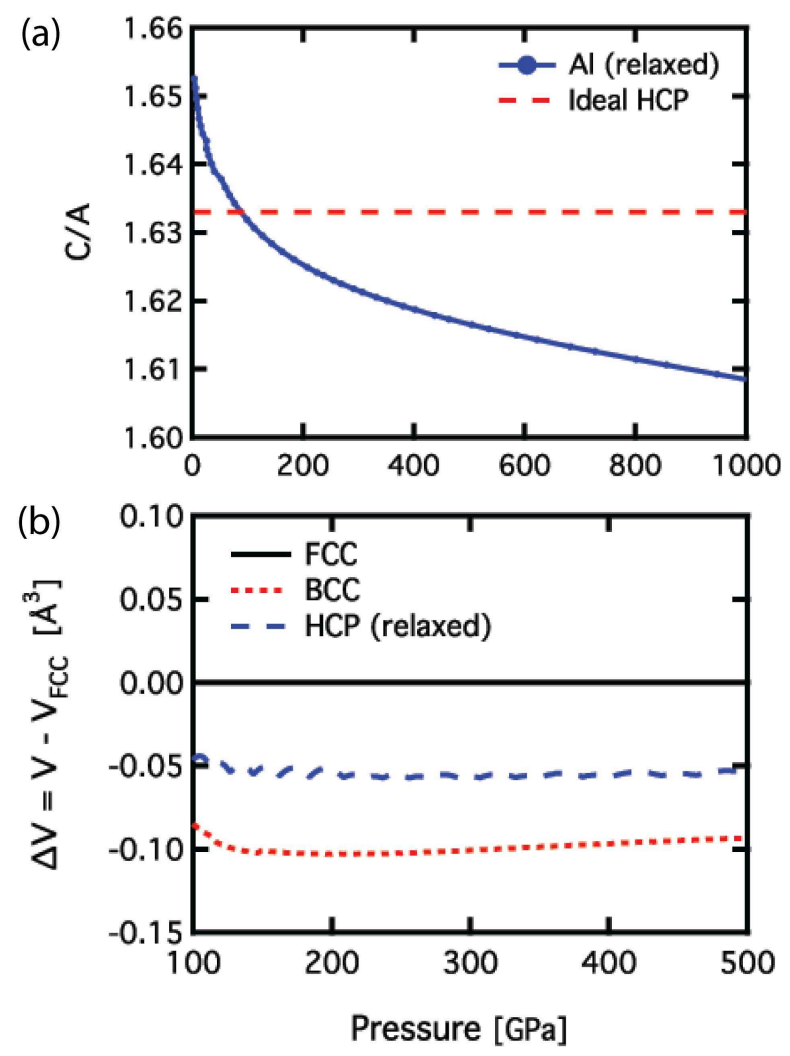

FIG. 2: (a) The c/a ratio of the hcp phase plotted with increasing pressure. (b) Volume versus pressure for the fcc, hcp, and bcc phases plotted relative to the fcc phase.

$2500 \mathrm{GPa}$. These results show that 1) there is little difference between the LDA and GGA predictions, hinting at a broad applicability of density-functional theory in either approximation, and 2) that the role of the core electrons starts to become noticeable only around 1200 $\mathrm{GPa}$, even if already at zero pressure the cores of the 3 e pseudopotential start to overlap ${ }^{27}$ The equations of state for aluminum in the fcc, bcc and hcp phase, using the 11e GGA pseudopotentials, are shown in Fig. 1 $\mathrm{b}$. Although the $11 \mathrm{e}$ and $3 \mathrm{e}$ calculations give consistent results up to $1200 \mathrm{GPa}$, the calculated transition pressures can vary, particularly for the $h c p \rightarrow b c c$ transition. This could easily derive from the fact that enthalpy differences between these three phases are only a few mRy (see Fig. 1b), and so, even at full computational convergence of all parameters, small effects (e.g. core-state relaxations), which could shift the calculated enthalpy by less than a mRy, can significantly affect the calculated transition pressures. On the other hand, core electrons seem to have a negligible effect in determining the equilibrium volume, bulk modulus, and even phonon dispersions (see below).

Our LDA and GGA results are consistent with previously reported GGA calculations; $; 11,12$ discrepancies arise with the LDA results reported in Ref. 10, that predict 


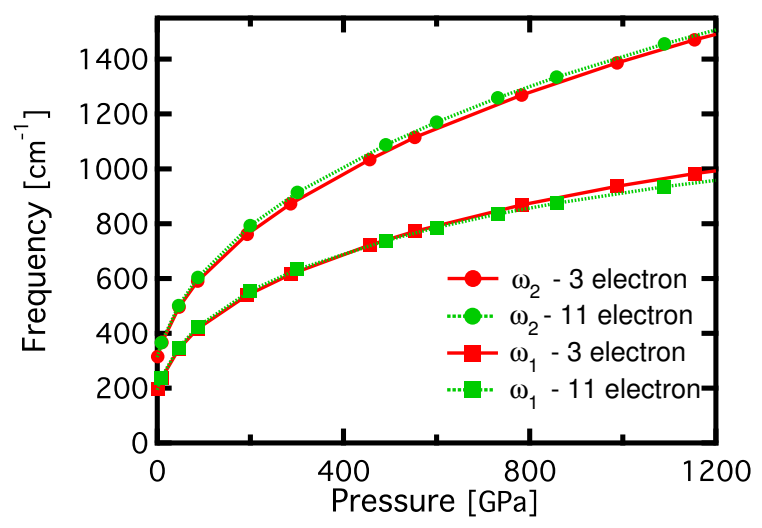

FIG. 3: The frequencies of the two non-degenerate acoustic phonon modes at X calculated with both the 3e and 11e pseudopotentials and plotted as a function of increasing pressure.

$205 \pm 20 \mathrm{GPa}$ and $565 \pm 60 \mathrm{GPa}$ for $f c c \rightarrow h c p$ and $h c p \rightarrow b c c$ transition pressures, respectively. This discrepancy could arise from Ref. 10 using only $10-15$ points to fit the equation of state: as reported there, this approximation could significantly affect transition pressures due to the aforementioned small enthalpy differences between competing structures. We also observe that all parameters of the calculation, and particularly the k-point sampling of the Brillouin zone, need to be carefully converged.

Although the pressure that we obtain for the $f c c \rightarrow$ hcp transition, $175 \mathrm{GPa}$, is consistent with previous works ${ }^{11.12}$, this result is lower than the experimental value of $217 \mathrm{GPa}$. As suggested in Refs. 11 and 12 this discrepancy could arise from excluding the phonon contribution to the free energy - a hypothesis that should be thoroughly tested, but that is beyond the scope of this brief report.

It should be noted that in our simulations the hcp phase was always fully relaxed to identify the optimal, equilibrium $c / a$ ratio; this is shown in Fig. 2 a. Comparison with experiment at $292 \mathrm{GPa}$ finds agreement in the c/a ratio to within $0.1 \%$ and well within experimental uncertainty 17 At $222 \mathrm{GPa}$ the predicted value of the c/a ratio differs from experiment by $1 \%$. Since experiments observe a region between 217 and $260 \mathrm{GPa}$ in which the fcc and hcp phases coexist, this discrepancy is made more reasonable considering that the system might be out of equilibrium. We also show in Fig. 2 $\mathrm{b}$ the equilibrium volumes for the different phases as a function of pressure. Volume differences between the phases are -0.055 , -0.104, and $-0.040 \AA^{3}$ for the $f c c \rightarrow h c p, f c c \rightarrow b c c$, and $h c p \rightarrow b c c$ transitions respectively, corresponding to volume changes of $0.6(6) \%, 1.4(0) \%$, and $0.6(1) \%$. We note that in our phase sequence, and in those discussed in the literature,,$\frac{7.8 .9}{.9}$ only the fcc, hcp, and bcc phases are considered. As a brief self-check we performed variable-cell relaxations at $1000 \mathrm{GPa}$ using a four-atom unit-cell and (a)

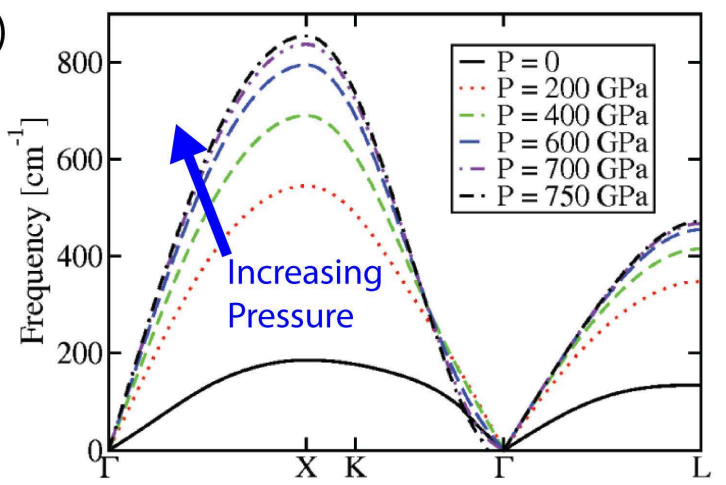

(b)

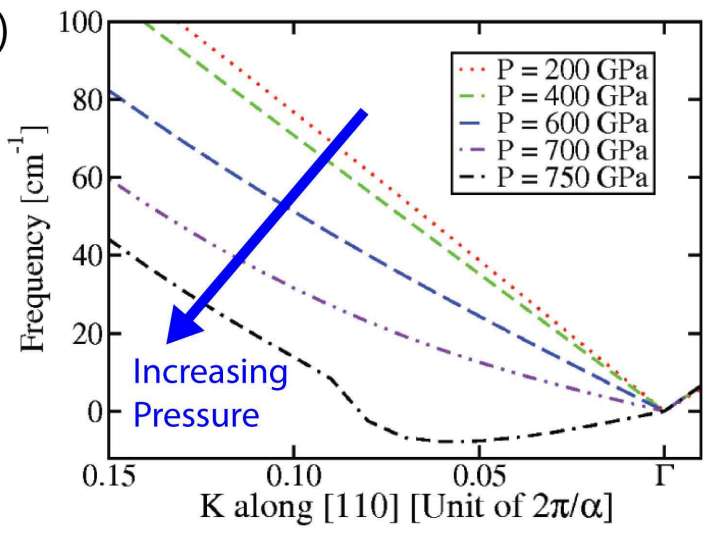

FIG. 4: (a) The lowest energy branch of phonon dispersion for fcc Al with increasing pressure. (b) As above, enlarged around $\Gamma$, in the $\mathrm{K} \rightarrow \Gamma$ direction. A steady flattening is observed with increasing pressure.

five random distinct perturbations. The bcc structure was always found.

In order to estimate the dynamical response of aluminum under compression we calculated both the phonon dispersions of the fcc phase and the cubic elastic constants (these were derived from the sound velocities, i.e. the slope of the phonons dispersions around $\Gamma$ ) as a function of pressure. Experiments have shown that the fcc phase may exist at pressures above the transition pressure either as a super-pressurized phase $\underline{\underline{16}}$ or as a two phase region: 17 Therefore the mechanical properties of fcc aluminum at pressures beyond the equilibrium transition pressure, and any mechanical instabilities that may lead to mechanical failure are relevant to high pressure experiments.

We calculated the phonon dispersions up to $1150 \mathrm{GPa}$ using DFPT. For aluminum this method has been shown to accurately reproduce experimental values at $\mathrm{P}=0 . \underline{24}$ Our calculations were performed with the 3e GGA pseudopotential, but compared at selected points in the Brillouin zone with 11e GGA calculations at pressures up to $1200 \mathrm{GPa}$. As shown in figure 3 the discrepancies between the $3 \mathrm{e}$ and $11 \mathrm{e}$ results are at most of the order of $3-4 \%$ at the highest pressure, and much smaller be- 


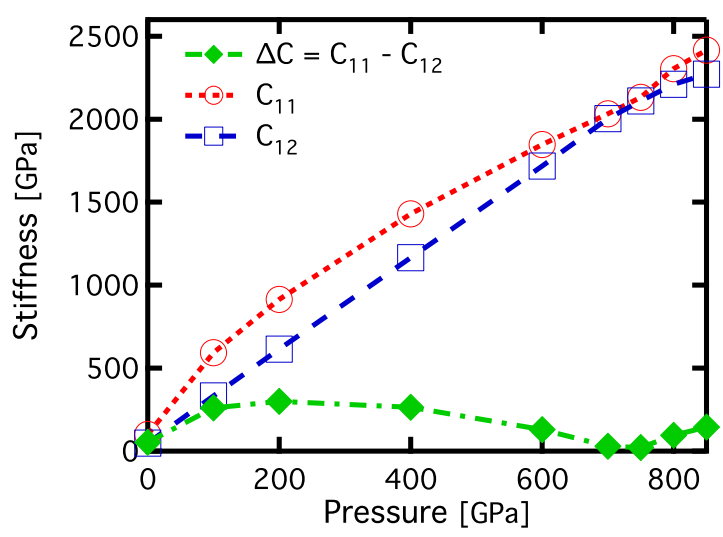

FIG. 5: Elastic constants as obtained from the sound velocities along the [100], [110], and [111] directions. According to the Born criterion the fcc phase becomes mechanically unstable when $\Delta c=c_{11}-c_{12}=0$; this occurs around $725 \mathrm{GPa}$.

low that. The phonon dispersions are shown in Fig. 4 a and we highlight the appearance, with increasing pressure, of a distinct softening of the lowest energy mode in the [110] direction. This starts to become evident at approximately $400 \mathrm{GPa}$, and is complete at $725 \mathrm{GPa}$, as highlighted in Fig. 4b. Since the slope of the dispersion curves is directly related to the elastic constants (Eq. 1), we can extract the stiffness tensor from the vibrational modes near $\Gamma$; in our case the Born ${ }^{28}$ criterion for stability is

$$
\frac{1}{2} m\left(\frac{1}{\hbar} \frac{\partial E}{\partial k_{110}}\right)^{2}=c_{11}-c_{12} \geq 0
$$

As Fig. [5] shows, the stiffness against shear deformation, $\Delta c=c_{11}-c_{12}$, decreases above $400 \mathrm{GPa}$ and goes to zero around $725 \mathrm{GPa}$, resulting in a Born ${ }^{28}$ instability. These results complement existing studies of the properties of bulk aluminum ${ }^{11,13}$ and suggest another shear failure mode, supporting previous studies suggesting shear failure modes to be inherent to bulk aluminum. 13,15 More advanced treatments of mechanical stability including such effects of anharmonic modes 24 and internal shear stresses created by loading $29,30,31$ need to be considered in relation to the specific experimental setup before reliable maximum stable pressures can be definitively determined.

Funding for this project has been provided by the U.S. Department of Energy Contract No. DE-FG0205ER46253. The authors would like to thank A. Dal Corso, I. Dabo, Y.-S. Lee, B. Wood, and J. Garg for useful discussions.
* Electronic address: marzari@mit.edu

1 O.L. Anderson, D. Isaak, and H. Oda, Rev. Geophys. 30, 57 (1992).

2 A. Alavi, M. Parrinello, and D. Frenkel, Science 269, 1252 (1995).

3 J.R. Cheeseman, G.W. Trucks, T.A. Keith, and M.J. Frisch, J. Chem. Phys. 104, 5497 (1996).

4 R.M. Martin, Nature 400, 117 (1999).

5 A. Heller, Science and Technology Review 4 July/August (1999); https://lasers.llnl.gov/

6 J. Edwards, K.T. Lorenz, B.A. Remington, S. Pollaine, J. Colvin, D. Braun, B.F. Lasinski, D. Reisman, J.M. McNaney, J.A. Greenough, R Wallace, H. Louis, D. Kalantar, Phys. Rev. Lett. 92, 075002 (2004).

7 J.C. Boettger and S.B. Trickey, Phys. Rev. B 29, 6434 (1984).

8 P.K. Lam and M.L. Cohen, Phys. Rev. B 27, 5986 (1983).

9 A.K. McMahan and J.A. Moriarty, Phys. Rev. B 27, 3235 (1983).

10 J.C. Boettger and S.B. Trickey, Phys. Rev. B 53, 3007 (1996).

11 G.V. Sin'ko and N.A. Smirnov, J. Phys.: Cond. Matt. 14, 6989 (2002).

12 F. Jona and P.M. Marcus, J. Phys.: Cond. Matt. 18, 10881 (2006).

13 W. Li and T. Wang, J. Phys.: Cond. Matt. 10, 9889 (1998).

14 N. Marzari, D. Vanderbilt, A. DeVita, and M.C. Payne, Phys. Rev. Lett. 82, 3296 (1999).

15 D.M. Clatterbuck, C.R. Krenn, M.L. Cohen, and
J.W. Morris, Phys. Rev. Lett. 91, 135501 (2003).

16 W.J. Nellis, J.A. Moriarty, A.C. Mitchell, M. Ross, R.G. Dandrea, N.W. Ashcroft, N.C. Holmes, and G.R. Gathers, Phys. Rev. Lett. 60, 1414 (1988).

17 Y. Akahama, M. Nishimura, K. Kinoshita, H. Kawamura, and Y. Ohishi, Phys. Rev. Lett. 96, 045505 (2006).

18 J.P. Perdew and A. Zunger, Phys. Rev. B 23, 5048 (1981).

19 J. P. Perdew, K. Burke, and M. Ernzerhof, Phys. Rev. Lett. 77, 3865 (1996).

20 P. Giannozzi et al., http://www.quantum-espresso.org

21 A. M. Rappe, K.M. Rabe, E. Kaxiras, and J. D. Joannopoulos, Phys. Rev. B 41, 1227 (1990).

22 D. Vanderbilt, Phys. Rev. B. 41, 7892 (1990).

23 F. Birch, Phys. Rev. 71, 809 (1947).

24 S. Baroni, S. de Gironcoli, A. Dal Corso, and P. Giannozzi, Rev. Mod. Phys. 73, 515 (2001).

25 T.L. Gilbert, Phys. Rev. B 12, 2111 (1975).

26 R. Stedman and G. Nilsson, Phys. Rev. 145, 492 (1966).

27 The core radius of the $3 \mathrm{e}$ pseudopotential is $1.43 \AA$; the half-bond length at $0 \mathrm{GPa}$ is also $1.43 \AA$, decreasing to $0.93 \AA$ at $1200 \mathrm{GPa}$. RRKJ ${ }^{21}$ pseudowavefunctions closely reproduce all-electron wavefunctions even for radii smaller than the matching radius, partially explaining the good results obtained with the $3 \mathrm{e}$ pseudopotential.

28 M. Born, Proc. Cambridge Philos. Soc. 36, 160 (1940).

29 R. Hill and F. Milstein, Phys. Rev. B 15, 3087 (1977).

30 J. Wang, J. Li, S. Yip, S. Phillpot, and D. Wolf, Phys. Rev. B 52, 12627 (1995).

31 F. Milstein, Handbook of Material Modeling, 1223 
(Springer, 2005). 